\title{
Integrated software and hardware system for power consumption and supply automated control in food industry enterprise
}

\author{
Serhii Baliuta, Liudmyla Kopylova, Iuliia Kuievda
}

National University of food Technologies, Kyiv, Ukraine

\section{Keywords: \\ Electric power \\ Control \\ Consumption \\ Power supply \\ Algorithm}

\section{Article history: \\ Received \\ 03.09.2018 \\ Received in revised \\ form 11.02.2019 \\ Accepted \\ 31.05.2019}

\section{Corresponding author:}

Iuliia Kuievda

E-mail:

julika@gmail.com

\section{DOI:}

$10.24263 / 2310-$

1008-2019-7-1-13

\section{Abstract}

Introduction. The research of power consumption and supply controlling process of the food industry enterprise (FIE) was conducted in order to increase the efficiency of transmission and use of electricity resources.

Materials and methods. The research is based on information systems design methods and modern technical equipment for automation of power system elements.

Results and discussion. To design the information structure of the control system an information data model is used, which is constructed according to the object-oriented principle.

The decomposition of the power supply and power management processes is performed then the control functions, the interaction between them and the users of the system are established.

The tasks of power consumption and supply control are realized with the help of the integrated software and hardware system (ISHS) of power consumption and supply automated control system in FIE (PCSACS FIE). The software part of the ISHS includes algorithms for solving the problems of operational control of power consumption and supply, the tools of system components interaction with the database and operating system (OS), as well as the graphical user interface of interaction of the information computation system with the power system dispatcher. Links between top-level PCSACS FIE devices are organized as Ethernet local area network using TCP/IP protocol with a transmission rate at least 10 Mbps. Communication channels between the upper PCSACS FIE level and the lower level controllers should be as a rule fiber-optic, providing absolute noise immunity. The design of the ISHS takes into account the conditions for the information security assurance.

Conclusions. The use of integrated software and hardware system created on the basis of information structure, developed in accordance with the UML methodology, ensures high efficiency of the power supply and consumption automated control system in the food industry enterprises. 


\section{Introduction}

The problem of development of automated control systems, that are used to ensure the reliability of electric power (EP) supply and regulatory norms for the EP quality indices along with the reduction of EP consumption, is relevant for the food industry, since it allows to increase the efficiency of generating capacities utilization and to reduce the energy intensity of products produced by enterprises. Adoption and implementation of adequate and effective management decisions for controlling the power consumption and supply of food industry enterprise (FIE) are provided on the basis of improving the structure, models and methods of management, the use of modern information technology and human-machine procedures.

We will consider scientific works devoted to the problems of software and hardware used in designing of automated control systems for power consumption and supply of industrial enterprises. Article [1] presents the structure and technical means of the automated system for registration of EP consumption "E1 - Energouchet". The system has two levels. At the lower level electronic counters "Euro Alpha" and "Alfa Plus" with digital communication channels are installed, and on the top level - modern computers with dispatcher's automated workplaces. The system is based on the client-server architecture and allows you to support the work of any number of client computers with dispatcher's automated workplaces. However, this system solves only the problem of monitoring the EP consumption.

The article [2] presents the software and technical means of the EP fiscal metering system made by the company "Energomera". The software, in the form of a set of software modules, allows you to organize the EP commercial metering of the power consumption objects. The set of technical means provides automation of the dispatcher's workplace, reports generation for creation of various forms of documents; data collection and database managing; determining the parameters of the devices of the electricity consumption metering system.

As a result of the analysis of works [1,2] it was established that the software and technical means represented in these works solve only the problems of the EP metering and do not allow to deal with problems of electric power consumption and supply automated control for industrial objects.

Recently, modern industrial controllers and SCADA systems are widely used in the design of automated power management systems [4]. The use of the listed above technical means allows to solve in real-time the problems of commercial metering of EP consumption, technical metering and monitoring of electrical loads of industrial enterperises, interaction of the operator with the information system, which is the basis for solving the problems of controlling the power consumption and supply of FIE.

Analysis of works [4-6] showed that the represented software and technical means provide only functions to control the electric power and the EP consumption. However, these means do not allow implementing the functions of monitoring the state of the electricity supply system, as well as standardization, planning, predicting and optimal control of power consumption and supply of industrial enterprises, which allow obtaining the main economic effect.

Objectives of research: to develop the information structure, software and to choose technical means for designing of an automated control system for power consumption and supply in the food industry enterprise on the basis of data collection and computer systems. 


\section{Materials and methods}

The process of managing power consumption and supply of FIE is studied, an information structure of the automated system, software algorithms are designed and the choice of technical means is carried out.

The research was conducted in the following order:

- a use case diagram has been constructed, in which the control system is represented by sets;

- the information structure of the automated control system of power consumption and supply of the FIE is developed;

- the software requirements are determined and the choice of technical means is made.

The UML methodology is used to build the information structure, which provides the development of representative models for the organization of interaction between the customer and the developer of information systems (IS) as well as different groups of IS developers, and also contains mechanisms for the expansion and specialization of basic concepts of language.

\section{Results and discussion}

Let us consider the features of power consumption and supply systems as control plants. Power supply and consumption systems of FIE are the most complex and important objects in comparison with other control plants. Their main features are as follows:

- at every moment electricity generation strictly corresponds to its consumption (there is no "electricity warehouse");

- in the case of failures, electrical transients are so fast that operational personnel can't prevent accidents propagation, without automatic control it is impossible to ensure reliable operating of the power supply system;

- failure of some element of the power consumption and supply system can lead to a complete disbalance of production, transmission, distribution and consumption of electricity and to the collapse of power system, as well as to the technological process violations, leading to enormous losses;

- returning to the normal operating mode can take a relatively long time; it is supposed that if within half an hour it wasn't possible to restore the normal mode, then the recovery period may be delayed for several days due to the batteries discharging and the loss of operational current when closing switches without the additional equipment is impossible.

Therefore, for power consumption and supply automated control system (PCSACS), it's significant to have high performance at the main levels of control, which is adequate to the speed of processes occurring in electric networks. At the PCSACS's lower level one uses specialized high-speed devices for powers system protection, oscillographic recording of fast transient failures and accidents propagations. At the upper level, one sets the minimum response time of the system and the fastest level of providing information. In order to find out the reason of failure, to analyze and compare the events and processes that occur in different parts of the power supply system, they need the same reference time. In addition, these processes must be time-bound with the events occurring in the power grid. Therefore, PCSACS provides the common timing system (CTS) for all controllers of the lower level, which are distributed over the enterprise's area. The accuracy of the binding of events to the CTS is determined by the high-speed processes taking place in the power consumption and 
supply system of the food industrial enterprise (PCSSFIE). CTS usually provides a temporal binding of the primary information, coming from the object, to the state scale of a common time with an accuracy of not less than $5-20 \mathrm{~ms}$. For PCSACS's equipment it's required high level of protection from electromagnetic interference (noise), since a significant part of the equipment is spatially located in zones which are prone to this effect. All devices and means of measurement must meet the special requirements for interference protection.

Taking into account the specifics of processes and different professional levels of the operational personnel of the dispatching control systems of mechanical and electrical processes, the complex objects require independent operator's workplaces. Territorially technical means of automated electrical and thermal-mechanical control systems are usually not combined. Digital power relay protection devices, reactive power compensation and power quality assurance devices, devices for communication with the object and sensors (current and voltage transformers, sensors of discrete signals, etc.). Electrical devices control systems are located in the substations of $0.4 \mathrm{kV}$ and switchgears of $6-220 \mathrm{kV}$ accessed only by specially trained electrotechnical personnel, on the other hand residual-current devices of technological automated control systems and their sensors (temperature, pressure, losses, etc.) as well as individual consumers of electric energy are placed in the premises of technological equipment who's access do not require special electrotechnical training.

\section{Features of PCSACS software structure}

These features are: the availability of optimization tasks of PCSSFIE's operating modes, power system reconfigurations, improving the EP quality, emergency protection automatics, emergency automated control for providing static and dynamic stability, remote change of set points, registration and storage of transient and emergency information, processing oscillograms with the symmetric components detection and the construction of vector diagrams, switching consumers-regulators (CR) etc. For these purposes, in addition to high response rate, there is a need for large amounts of memory in control devices and the specialized centralized data store, on the basis of which these tasks could be solved. In PCSACS design one also takes into account the special aspects of maintenance of electrical devices and climatic conditions of PCSSFIE.

PCSACS combines the above-mentioned control systems of individual electrotechnical objects, automation, control and protection devices into a single information and control system, which enables operating personnel to control the operating modes of PCSSFIE and external power supply system. It should be taken into account that these devices and automatics solve the problems of local control of individual objects, relatively independent, geographically they can be in different parts of the PCSSFIE and provide the implementation of a single task ensuring the functioning of the PCSSFIE, coordinated with each other in accordance with the principles of operation, control algorithms and parameters triggering The autonomy of these devices is the main principle of the reliability of their operation in normal, emergency and post-failure modes.

\section{Algorithms and methods of power consumption and supply control in FIE}

To ensure the effective operation of PCSACS it is necessary to perform an analysis of PCSSFIE: the functions that ensure system's operating modes and the interaction between the automated control system (ACS) and the system's users. These functions are: gathering information and verifying it for reliability; monitoring of the technical state of the electric network; interactive interface of the power system dispatcher with the information and 
computing system (ICS), which provides visualization of the issued recommendations and immediate reasonable decision-making process for power consumption and supply problems in real-time mode.

For the construction of ready-made solutions in PCSACS there is a subsystem of control procedure selecting, which is supposed to be a functional block. Solutions, formed by the automated subsystem of control procedure selecting, are complete, on their basis one can uniquely make corresponding management decisions. However, the final decision on choosing the control command, depending on the situation, can be made by the dispatcher. This is due to the fact that by no means always quantitative analysis of the situation provides the only correct solution.

It should also be noted that effective control of power consumption and supply in FIE implies the availability to the dispatcher of necessary information about the current and predicted state of the PCSSFIE. This requirement leads to the need to monitor the production cycle parameters. This does not mean the need to create an additional system of data collection, since in many cases, monitoring of technological processes already exists and it is only necessary to obtain the necessary data from the corresponding automated control system (ACS) service server of the technological process or from the enterprise's ACS server.

In the studied system the users are (Figure 1):

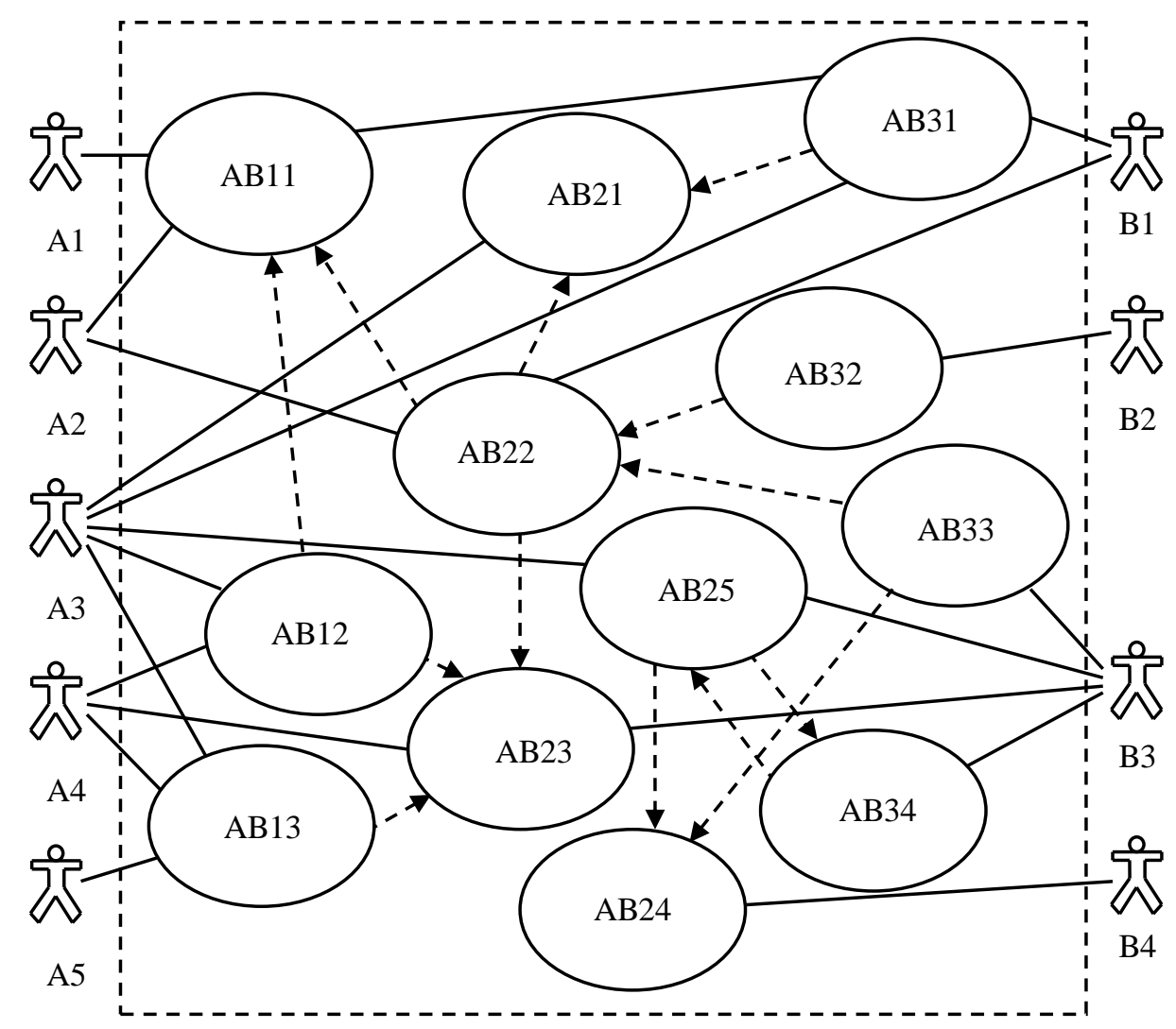

Figure 1. The Use Case Diagram of power consumption and supply control system 
A1: Control Plant (CP). A control plant of power consumption and supply is the electric network of FIE and electrical devices.

A2: Receiving and transmitting devices (RTD). These are tools used for polling sensors and getting their data. Industrial controllers, digital relay protection and specialized hardware devices for data collecting and transmitting, all they serve as RTD.

A3: Information and Computing System (ICS). The system, which implements a set of mathematical methods and software tools for their implementation, aimed at solving the tasks of controlling the power consumption and supply of FIE.

A4: Power system dispatcher. He is a member of Chief Power Engineer's Department (CPED), who interactively manages the power consumption and supply of the FIE in the realtime mode interacting with ICS.

A5: Application specific workstations (ASW). These workstations are a set of hardware and software tools for the employee to fulfill their official duties.

B1: Power consumption and supply control database of FIE. The database is a source of data. It stores the data of meters and sensors, which is necessary for PCSACS tasks completing.

B2: Technological Department Dispatcher (TDD). The specialist who monitors the production process and performs in real-time mode control of repairing and restoration tasks on the enterprise's power system.

B3: Electrical engineer. He is a specialist of CPED, which controls the work of all users.

B4: Chief Power Engineer. Its function includes interaction with other structural subdivisions of the enterprise and external organizations.

Functions performed by PCSACS of FIE:

AB11: Registration of measured data. For the registration of telemetering data, polling of meters and sensors at predetermined time intervals and transmission of their reading to ICS are carried out.

AB12: Monitoring of technical condition. The main regulatory duty of the power system dispatcher is to control the operation of the FIE's power system and its consumers. Using the data stored in the database, as well as the recommendations of the ICS, CPED staff ASWs receive the relevant data on the status of controlled system elements. Based on it, a conclusion about the quality of the power system operation is made.

AB13: Displaying operational data about the status of PCSSFIE. With the help of the interface, the ICS translates data from the database to CPED staff ASWs.

AB21: Data verification. Efficiency of power consumption and supply control of FIE is largely determined by the reliability and quality of data coming from measuring devices. Therefore, a separate task is to ensure the reliability of the measurement data - the detection of error (abnormal) measurements of controlled quantities. This task is an integral part of any control system, since unresolved gross errors in measurements determine the reliability of the data used to make control decisions.

AB22: Searching for solutions for operational-dispatch control (ODK) tasks. The main ODK tasks are operative restoration of FIE's power supply and normal parameters of released electricity; creation of the most reliable post-failure scheme and operating modes of FIE's power system; determination of the state of disconnected electrical equipment and restoration of its electricity supply; switching on and off consumers-regulators to maintain rational levels of power consumption. This is done by reconfiguring the FIE's power system by changing the position of the switches and redistributing the load between power consumers.

AB23: Choosing a network topology and selecting control actions. This function describes a possibility of choosing a new topology of the FIE's power system in cases of 


\section{— Processes, Equipment and Control Systems -}

emergency situations as well as during power equipment repairing period and power shortages in external power system.

AB24: Determining of the power consumption level declared by FIE. Every day, a summary of power consumption indicators of by production units are consolidated: actual and planned absolute and specific power consumption, coefficients of use of the established limits, cost rates. On the basis of this data, decisions are made to maintain the power consumption parameters of FIE within the specified limits. ODK decisions preparing is carried out using the decision support subsystem.

AB25: Predicting and normalizing electrical loads. Proceeding from the tendency of changes in power consumption and quantity of released products, the prediction of electrical loads and the normalization of power consumption are carried out at the power system nodes, to which separate technological facilities are connected.

AB31: Maintaining of the system's database. For efficient control of power consumption and supply electricity of FIE in real-time mode, an information database on electric power system is filled and maintained in the actual state. Its main functions are the execution of tasks scheduled by the administrator and transfering of data to remote clients ASW (Chief Power Engineer ASW, Electrical Engineer ASW, Power System Dispatcher ASW, etc.).

AB32: Prohibiting performing control actions (CA). In the event that the actions of the Power System Dispatcher to change the position of the switches and disconnect the electrical receivers contradict the schedule of planned repairs, the Chief Dispatcher shall prohibit the implementation of such actions.

AB33: Determining of power supply modes indicators.

AB34: Determining of power consumption modes indicators. The operating of the power system and of the electric consumers, as an integral part of PCSSFIE, is characterized by a set of indicators whose values determine the operating mode of the power system and consumers in time. Quantitative indicators of the operating mode are the values of electric energy, power, voltage, current, EP quality indices and other parameters determined by the tasks solved by the system.

On the basis of the Use Case Diagram, the information structure of PCSACS in Data Flow Diagram (DFD) notation was developed (Figure 2). 


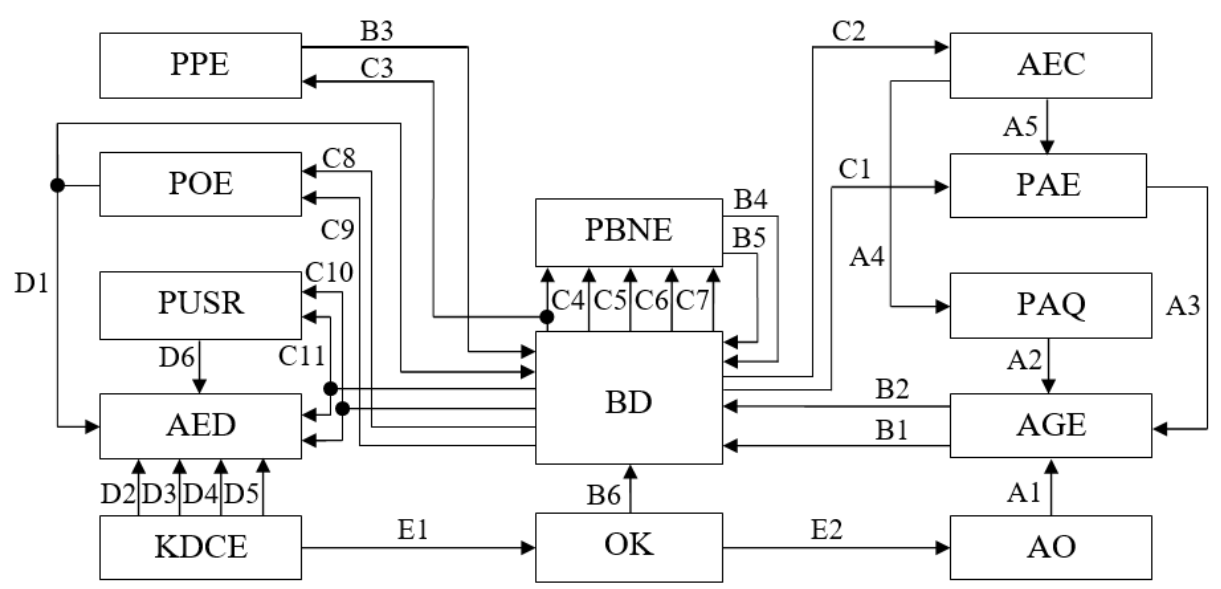

Figure 2. Information structure of PCSACS

Functioning of the information system is provided by the following users and subsystems: OK - control plant (PCSSFIE); AO - Production Department Dispatcher ASW; AGE - Chief Power Engineer ASW; AEC - electrical department ASW; AED - Power System Dispatcher ASW; PAQ - subsystem (module) of power consumption modes analysis; PAE - subsystem (module) of power supply modes analysis; BD - PCSACS database, PBNE - subsystem (module) for calculating balances, norms of EP expenses; PPE - subsystem (module) for predicting EP consumption; POE - subsystem of power supply modes optimization; PUSR - selecting subsystem SR and power consumption optimization; KDCE - generating subsystem of control actions for FIE power consumption and supply control.

The following Data Flows are used for control actions generating and decision making: A1 - limitations on the power supply system (PSS) topology and of the CR list; A2 - cost plan and CR list, A3 - decisions to ensure normative values of EP quality indices (EPQI), optimal modes and PSS topology; A4 - data concerning power consumption and CP selecting; A5 - data on PSS modes and decisions; B1 - approved decisions on the normalization of EPQI and the PSS topology selecting; B2 - approved EP and CR cost plan; B3 - estimated EP values; B4 - balances and EP consumption rates; B5 - list of enabled CRs; B6 - data concerning the control plant status; $\mathrm{C} 1$ - balance and expenses of EP, actual and predicted power consumption levels and CRs; $\mathrm{C} 2$ - operational data on the state of the PSS; C3 - actual costs of EP; C4 - decisions taken on CRs; C5 - data on production output; C6 actual costs of EP and EP limits; C7 - approved EP schedule; C8 - PSS mode parameters; C9 - list of disconnected CRs; C10 - recommended topology of PSS; C11 - approved levels of EPQI. As a result of system operating, the following control actions are formed: D1 control actions for PSS optimizing; D2 - EPQI controlling decisions; D3 - prohibiting performing control actions; D4 - decision on PSS topology; D5 - made decisions on power system management; E1 - control actions transmitted to the control plant; E2 - information required for selecting the CR and PSS topology. 


\section{Application specific workstations (ASW)}

PCSACS includes ASWs of Administrator, Chief Power Engineer, Power System Engineer, Power System Dispatcher, Equipment Repair Engineer and Economist, which include a set of functions assigned to them.

Administrator's ASW should provide: access to the configuration of the logical scheme of the control system; creating and editing of database queries used by other ASWs; data exchange between databases and Microsoft Office programs; maintaining a system event log and accessing its records.

Power System Engineer's ASW should provide: analysis of the company's power consumption, calculation of power consumption and power balance; choosing optimal control parameters of FIE power consumption.

Chief Power Engineer's ASW should provide: on demand displaying of the necessary current and reporting information about the company's power supply.

Power System Dispatcher's ASW should provide: displaying electric schematic diagram with state of switching equipment; displaying current and summary expenses of electric power for power consumption units; displaying of daily charts of power consumption by power consumption units in tabular and graphic form; displaying the enterprise's power consumption predicted values and warning about possible exceeding of the established limits; FIE power supply control on the basis of human-machine dialogue in real-time mode.

Electrical Equipment Repair Engineer's ASW should provide: making and displaying of the repairing schedule for electrical equipment; displaying data on demand about the current technical condition of electrical equipment.

Power Management Engineer's ASW should provide: implementation of the power consumption limits regulated by the power supplying company; analysis of the enterprise's power consumption in economic terms, obtaining necessary reports and transferring information to other departments of the enterprise.

The hierarchical structure of the information control system is presented in Figure 3. To provide the functioning of the information system, an integrated software and hardware system (ISHS), consisting of hardware and software components, has been developed.

\section{Software part of ISHS}

The main element of this part of ISHS is the information and computing system (ICS), which includes software modules that implement algorithms for solving power consumption and supply control tasks, the means of interaction with the enterprise power system database and operating system (OS), as well as the IEC graphic user interface for a dispatcher, a power system engineer, an power management engineer, a chief power engineer. To solve the above-mentioned tasks, the following software modules (subsystems) are supposed:

- module of digital relay protection and emergency automatics terminals (DRPA);

- I/O module for data which is not transmitted through the DRPA terminals;

- precise astronomical time input module;

- module of the central (common for generating units) and local (in area of the switchgear) alarm;

- $\quad$ module for power supply system control;

- module for electric power quality control in PSS;

- module of automated configuration of power supply system;

- module of automated control of FIE power consumption. 


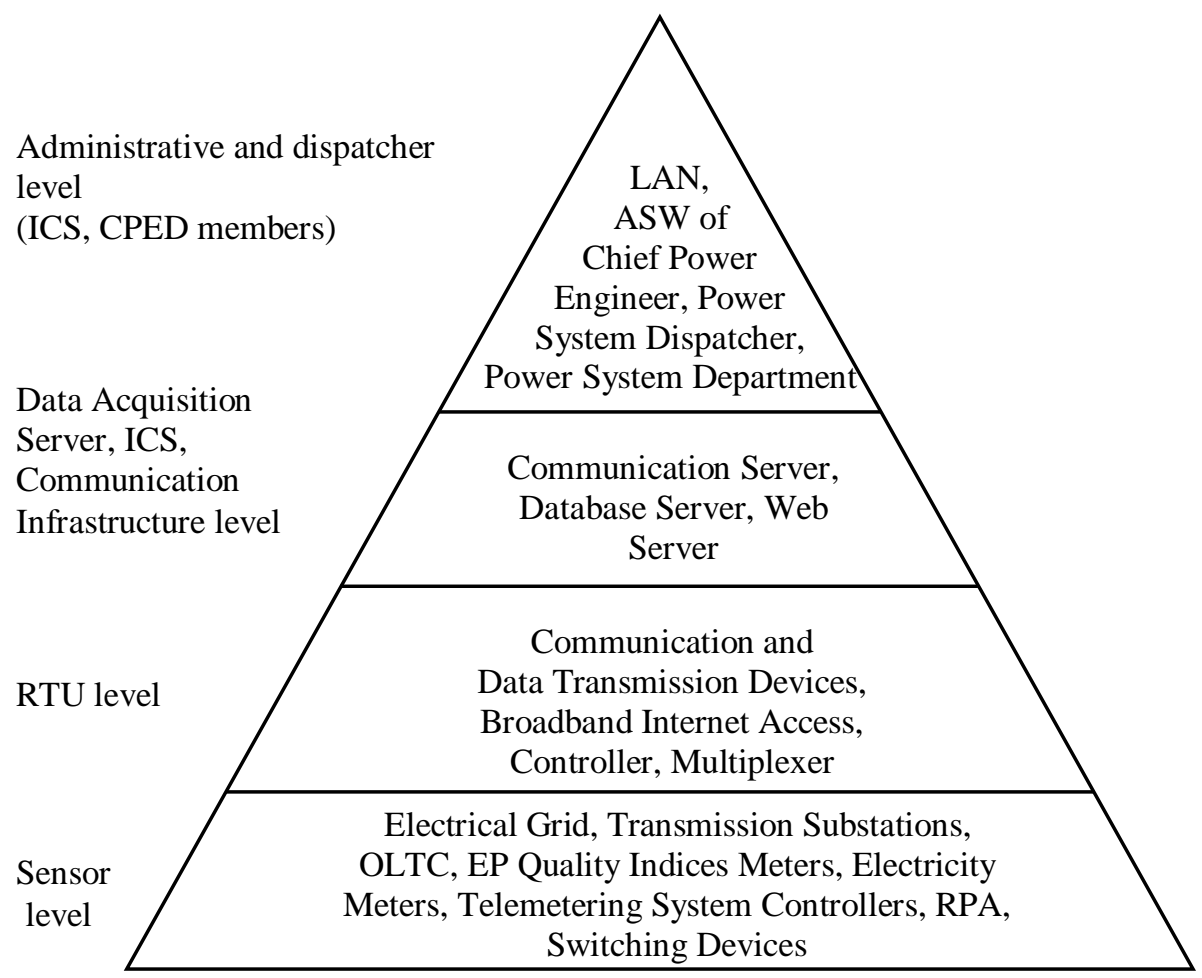

Figure 3. Hierarchical structure of information system of power consumption and power supply management

\section{Hardware part of ISHS}

The hardware part of ISHS is based on a local area network (LAN). It's a distributed system, which includes geographically remote objects. The main source of data in PCSACS is the multifunctional digital relay protection and automatics of power supply system (DRPA terminals), which are at the same time devices of the PCSACS lower level. In addition, remote control units for electrical equipment (RCU) may be used. They provide the collecting and transferring to operator's level about $90 \%$ of data necessary for the PSS operational control. Naturally, PCSACS is constructed, as a rule, on the basis of serial specialized control systems of the manufacturer of digital terminals.

The data received from DRPA terminals is used on workstations to form the mimic diagram of the switchgear, display current parameters, control and other purposes. However, when the feeder is out of service, when there is no operational current, the terminal stops transmitting data. Therefore, in order to obtain on the workstation's screen the image of circuit breaker under repair, the disconnectors and grounding blades position, the data from the block contacts of these devices is transmitted through the remote terminal units (RTU), which have independent operational current.

Power supply of RTU and connected circuits is organized using a separate circuit breaker, since it should not depend on the feeder operational current. The data from DRPA, RTU and ACS is transmitted via digital fiber-optic channels to the server that takes a central place in the PCSACS. The server has connection channels to the lower level of control, 
operators' workstations (computers), the common time system, automated system of energy resource technical registration and the router.

The main technology control system and dispatcher control system are connected to PCSACS through a router, which connects these different networks and forwards data packets between them.

\section{General principles of data exchange organization in ISHS}

Data flow from electrical devices to workstations, adjacent and higher level systems, as well as control commands in reverse direction, is carried out using lower and upper level LAN.

The lower level LAN transmits data from electrical devices to the server and the control commands in the reverse direction.

Connection of electrical devices to the lower level LAN is carried out through the data communication ports available in the DRPA devices, local automation systems and in RTU. The upper level LAN transmits information from the server to PCSACS workstations, adjacent and higher level systems, as well as control commands in the reverse direction. Requests for server data and sending control commands are performed by workstations called clients, and the structure of network architecture - client-server or dedicated server. For the data exchange in the networks of the lower and upper levels PCSACS uses the physical communication channels through which the data is transmitted in the form of electric, optical or radio signals (electrical or optical cables, radio or satellite communication channels). Data transmission is carried out according to certain rules, defined in data communication protocols. Data communication protocols are implemented in network software.

\section{Organization of data transfer to ISHS}

For data transmission, communication lines of RS-485, RS-422, RS-232C interfaces are used. Depending on the used interface only the electrical parameters of transmitters, receivers, as well as the construction of communication lines will be different. In these lines, data is transmitted in packets ranging from tens to several hundred bytes. It uses an asynchronous serial start/stop data transmission in which the bytes of each packet are transmitted sequentially one after another, bit per bit. In the case of asynchronous data transmission, there is no special line for synchronization of the transmitter and the receiver. The data to be transmitted is the control commands; signals characterizing the state of the equipment and its operating mode; emergency and warning alarms; measured values of currents, voltages, frequencies; other technological parameters and discrete signals. The software in DRPA, RTU, controllers, and other devices runs in a loop that can only be affected by extraordinary events, such as the appearance of device events requiring immediate processing and causing program loop interruptions. The program loop can range from one to hundreds of milliseconds.

Connection of DRPA terminals, electronic meters and security terminals is provided through high-speed interfaces. High speeds of data transmission and processing allow the same interfaces and data communication protocols to be used at the lower and upper levels of the ACS, which simplifies its structure. On the other hand, the modern development of telecommunication technologies allows designing highly reliable networks due to the use of duplicated communication channels and specialized protocols for relay protection Modbus, TCP/IP, IEC 61850 and others, reserve the Ethernet network star topology. Consider the option of the PCSACS structure, where at the lower level ABB's REX 600-th series terminals 
(RET 615, REF 620, REM 620, etc.) are installed. These terminals can be equipped with an additional communication module that has two independent optical Fast Ethernet ports (100Base-FX interface). For working in Fast Ethernet networks terminals include protocols IEC 61850, DNP3, TCP/IP. Using these communication modules, one can build a completely redundant network for the "star" or "ring" topology.

In the redundant network of "star" topology there are two Ethernet switches. Each switchboard is connected to one optical port of the communication modules of all DRPA terminals. On the PCSACS server, two network adapters are installed, each of which connects to the corresponding switch. In fact, such a network consists of two independent networks. If one of the networks fails, data transmission will continue in the second network with zero switching time. The main advantage of such a scheme is its high reliability through $100 \%$ redundancy, network independence of state of the individual terminals, which switching off or malfunction does not affect the rest of the network. As disadvantage of this scheme one can consider the need to lay a large number of cables - two from each terminal to the switches. It should also be noted that, since there are no direct links between the terminals, the GOOSE messages will be transmitted through an additional element - the switch.

\section{SCADA systems specifications}

SCADA systems are the basis of operational-dispatching control systems of power consumption and supply operating modes.

To create Windows-like user interfaces, communications with meters and actuators, SCADA-based systems on the MS Windows platform are used. They contains standard data interfaces and API functions that facilitate the development of individual control subsystems and their integration into power system ACS of the enterprises' ICS (integration with ERPsystems SAP, "Galaxy", etc.). The software is developed using both built-in languages of SCADA systems or languages that allow designing interface to them, using the known CASE-tools and the universal modeling language UML (Rational Rose and ARIS), what greatly simplifies the process and reduces design time of control system.

When developing the software it's reasonable to use existing, ready-made COTS (Commercial Off-The-Shelf) problem-oriented tools.

An approximate list of criteria for evaluating SCADA systems can be conventionally divided into three large groups of indicators:

- specifications;

- cost characteristics;

- performance characteristics.

Hardware platforms for SCADA systems. The analysis of the list of such platforms is necessary, since it decides the question whether the implementation of this SCADA system is possible on the available computing tools, as well as assessment of the cost of system's technical support (being developed in one operating environment, the application can be implemented in any that supports the selected SCADA package). In different SCADA systems, this issue is solved in different ways.

At the same time, such SCADA systems as RealFlex and Sitex accept as the basis for software only one operating system - real-time OS QNX.

The vast majority of SCADA-systems are implemented on MS Windows platforms. These are such systems that offer the most complete and easy to upgrade MMI - tools. 
Tools of SCADA-systems network support. Obviously, for the efficient functioning in this heterogeneous environment, the SCADA system should provide a high level of network service. It is desirable that it should support standard network environments (ARCNET, ETHERNET, etc.) using standard protocols (NETBIOS, TCP/IP, etc.), and also should provide support for the most popular network standards from the class of industrial interfaces (PROFIBUS, CANBUS, LON, MODBUS, etc.) These requirements anyway satisfy practically all of the SCADA systems considered, with the only difference that the set of supported network interfaces, of course, is different.

Built-in command languages. Most SCADA systems have built-in high-level languages, VBasic-like languages, which allow you to generate an adequate response to events associated with changing the value of a variable, with a certain logical condition, with a keystroke, as well as with the execution of some fragment with given frequency relative to the whole application or a separate window.

Database support. One of the main tasks of dispatch and control systems is the data processing: collection, real time analysis, storage, compression, forwarding, etc. Thus, in the system to be created, there should be a database.

Almost all SCADA systems, including Genesis, InTouch, and Citect, use the ANSI SQL syntax that is independent of the database type. Thus, applications are virtually isolated, which allows you to modify the database without seriously changing the application itself, create independent software for analyzing information, use the already developed software, focused on data processing.

Graphic features. Functionally graphical interfaces of SCADA-systems are very similar. Each of them has a graphical object-oriented editor with a certain set of animation functions. The vector graphic makes it possible to perform a wide range of operations on the selected object, as well as to quickly update the image on the screen using the animation tools.

It is also extremely important to support the GUI (Graphic Users Interface) in these systems. Since most of the SCADA systems are running under Windows, it determines the type of GUI used.

Openness of systems. The system is open, if it identifies and describes the data formats used and the procedural interface, allowing it to connect to the "external", independently developed components.

Development of own software modules. In the face of the automation system developers, the question often arises about the creation of their own (not provided within the framework of SCADA systems) software modules and their inclusion in the created automation system. Therefore, the issue of system openness is an important characteristic of SCADA systems. In fact, the openness of the system means the availability of system specification calls (in the sense of SCADA) that implement one or another system service. It can also be access to graphic functions, functions of operating with databases, etc.

I/O drivers. Modern SCADA systems don't restrict the choice of lower-level hardware, since they provide a large set of drivers or I/O servers and have well-developed means for creating their own software modules or drivers for new lower-level devices. The drivers themselves are developed using standard programming languages. The question, however, is 
whether the SCADA-engineer needs only the specifications for access to the system kernel supplied by the firm-developer in the standard set (Trace Mode), or he requires for the creation of drivers special packages (FactoryLink, InTouch), or, in general, developing drivers must be ordered from the developer company.

The development of a control system using SCADA systems includes the following steps:

- Development of the automation system architecture as a whole. At this stage, the functional purpose of each node of the automation system is determined.

- Solving issues related to the possible support of a distributed architecture, the need to create hot node backups, etc.

- Creating an applied control system for each node. At this stage, a specialist in the field of automated processes provides the nodes of architecture with algorithms, the totality of which allows you to solve automation tasks.

- Matching the application system parameters with the information exchanged between lower-level devices (for example, programmable logic controllers - PLC) with the outside world (processor sensors, actuators, etc.).

- Setup of the created application in the mode of emulation.

\section{Technical means for power supply system automation}

Currently, modern protection, automation and control microprocessor devices (RPA terminals) of various primary electrical equipment are widely implemented at all voltage levels of $0.4-110 \mathrm{kV}$ and above. Terminals are used in secondary switching circuits for use as primary and back-up protections.

Complete devices of protection, control and automatics. Simultaneously with the implementation of the relay protection functions, RTU-terminals are lower level interface devices in control systems. Terminals can transmit measurable values, parameters of emergency modes, set point values, oscillograms, the equipment state in the control system, and also perform remote control of the control plant. Integration of relay protection terminals into the ACS allows reducing the capital costs of the RTU equipment when creating the power supply ACS.

Terminals of protection and control are designed on a microprocessor element base for protection of various primary electrical equipment. Terminals are used in circuits of secondary switching for use as main and reserve protection with voltage $0.4-35 \mathrm{kV}$.

Process controllers and telemechanics devices are used as controllers and easy to adapt to different transmission environments and traffic modes. Terminals have a modular structure and can be used on objects with a number of signals from 20 to 1800 . Terminals allow flexible programming of collection, primary processing and transmission of data and provide execution of local automation functions.

Alarm devices are intended for organization of the local or central signaling system of the power unit.

The device displays on the local panel a group or individual alarm, it manages an audible alarm. It is equipped with a communication port for connecting to the ACS a portable electrician ASW (relay) to work with protections and an event printer. The standard protocols ANSI and MODBUS are used for the exchange of upper level control systems. 
The device for receiving and transmitting data (DRTD) is intended to provide the functioning of the local automated metering system the enterprise and performs the following functions:

- collection of measurements from digital and pulse outputs of meters;

- calculation of the named parameters of electric power;

- maintaining archives;

- support for communication with local ACS and remote ASW Energosbut.

The device performs functions of collecting, pre-processing and storing data.

\section{Electricity metering and control systems}

For technical and commercial electricity metering, installed counters with pulsed outputs or intelligent digital counters (for example, "Alpha") are used. Intelligent counters perform the following functions: electricity metering for 4 tariffs, 4 seasons, 4 types of weeks with an accuracy class of 0.2 and 0.5 ; measurement of active and reactive power in two directions; power quality control; load schedule storage; transmission of measurement results via digital and pulse channels.

To store electrical quantities analog values in the system special devices can be used that combine the functions of sensors and control the quality of electric power.

The system should provide the following system-wide functions: maintenance of a single system-wide database; system administration with the availability of different levels of access, ensuring access control of database users; software integration with existing system development tools; configuration settings for the system menu for ASWs; the maintenance of a system log of events.

The implemented software system solutions should provide the opportunity to further increase the number of control plants, types of energy sources, types of information analysis and number of ASWs, as well as integration of the system with other ACS subsystems.

\section{Conclusions}

The use of the UML methodology to analyze the power consumption and supply control process of FIE allows identifying the users of the control system and the basic control functions. The design of the information structure is performed according to the DFD methodology, which reflects the main data flow that provides control of power consumption and supply. To implement the control functions the integrated software and hardware system is developed. It consists of modules that ensure the operation of PCSACS. For data transmission communication lines are utilized, which are built using interfaces such as RS485, RS-422, RS-232C. The SCADA systems are the basis of power consumption and supply modes operational-dispatcher control systems. The technical means of PCSACS are modern microprocessor protection, automation and control devices (relay protection terminals) of various primary electrical equipment of all levels of voltage $0.4-110 \mathrm{kV}$ and above.

\section{References}

1. Bulaev Iu.V., Tabakov V.A., Eskin V.V. (2001), Kompleksnaia avtomatizatsiia energosnabzheniia predpriiatiia, Promyshlennaia energetika, 2, pp. 11-15.

2. Mirzoian Iu. T. (2000), Programmnoe obespechenie KTS «Energomera», Energetik, 8, pp. $42-44$. 


\section{- Processes, Equipment and Control Systems-}

3. Kapitonova B. Tuganov V. Satarov L. (1996), Territorialno-raspredelennaia avtomatizirovannaia sistema ucheta i kontrolia elektropotrebleniia, Sovremennye tekhnologii avtomatizatsii, 1, pp. 78-80.

4. Egorov V.A. (2001), ASKUE sovremennogo predpriiatiia, Energetik, 12, pp. 41.

5. Kovezev S. N., Urazov B.V., Chumakov V.V. (2001), Sozdanie ASKUE na baze IVK «Sprut», Energetik, 2, pp. 11-13.

6. Molokan E. (1996), Avtomatizatsiia ucheta energopotrebleniia, Sovremennye tekhnologii avtomatizatsii, 1, pp. 74-76.

7. Cheremisin M. M., Kholod A. V. (2012), Kompleksna avtomatyzatsiia enerhoobiektiv na bazi suchasnykh SCADA system, Visnyk Vinnytskoho politekhnichnoho instytutu, 3, pp. 128-131.

8. Prakhovnik A.V., Rozen V.P., Degtiarev V.V. (1985), Energosberegaiushchie rezhimy elektrosnabzheniia gornodobyvaiushchikh predpriiatii, Nedra, Moscow.

9. Rezchikov A.F., Ivashchenko V.A. (2008), Upravlenie elektropotrebleniem promyshlennykh predpriiatii, Saratov.

10. Zamulko Anatoly, Veremiichuk Yurii (2014), Methods of controlling power consumption in terms of reforming market conditions, Scientific Journal of Riga Technical University Power and Electrical Engineering, 32, pp. 41-45.

11. Auffhammer M., Blumstein C. (2007), Demand-Side management and Energy Efficiency revisited, Berkeley.

12. Steimle W., W. Thoma, Wille-Haussmann B. (2006), Intelligent Energy Management in Low Voltage Grids with Distributed Resources, IEEE Transactions on Power Systems, pp.125-135.

13. Choi J.H., Kim J.C. (2001), Advanced voltage regulation method of power distribution systems interconnected with dispersed storage and generation systems (revised); IEEE Transactions on Power Delivery, 16(2), pp. 329-334.

14. Liu Y., Zhang P., Qiu X. (2000), Optimal reactive power and voltage control for radial distribution systems, IEEE Power Engineering Society Summer Meeting, pp. 85-90.

15. Wasiak M., Thoma C., Foote R., Mienski R., Pawelek P., Gburczyk G., Burt A., Morini (2006), A Power Quality Management Algorithm for Low Voltage Grids with Distributed Resources, IEEE Transactions on Power Delivery.

16. Lopes M.A.R., Antunes C.H., Martins N. (2012), Energy behaviours as promoters of energy efficiency: A 21st century review, Renewable and Sustainable Energy Reviews, 16(6), pp. 4095-4104.

17. Ralf Martin, Mirabelle Muûls, Laure B. de Preux, Ulrich J. Wagner (2012), Anatomy of a paradox: Management practices, organizational structure and energy efficiency, Journal of Environmental Economics and Management, 63(2), pp. 208-223

18. Lässig J., Riesner W. (2012), Energy efficiency benchmark for industrial SME, Smart Grid Technology, Economics and Policies (SG-TEP), 2012 International Conference, pp. 1-4. 